\title{
Overexpression of anti-fibrotic factors ameliorates anti-fibrotic properties of Wharton's jelly derived mesenchymal stem cells under oxidative damage
}

\author{
Natakarn Nimsanor ${ }^{1}$, Jitrada Phetfong ${ }^{2}$, Narisorn Kitiyanant ${ }^{3}$, Witchayapon Kamprom ${ }^{4}$, \\ Aungkura Supokawej ${ }^{1, *}$ \\ ${ }^{1}$ Department of Clinical Microscopy, Faculty of Medical Technology, Mahidol University, Salaya, Nakhon Pathom, Thailand; \\ ${ }^{2}$ Center for Research and Innovation, Faculty of Medical Technology, Mahidol University, Salaya, Nakhon Pathom, Thailand; \\ ${ }^{3}$ Stem Cell Research Group, Institute of Molecular Biosciences, Mahidol University, Salaya, Nakhon Pathom, Thailand; \\ ${ }^{4}$ Department of Clinical Microbiology and Applied Technology, Faculty of Medical Technology, Mahidol University, Salaya, \\ Nakhon Pathom, Thailand.
}

\begin{abstract}
Summary $\quad$ Transplantation with Wharton's jelly derived mesenchymal stem cells (WJ-MSCs) showed great benefits for restoring myocardial function. However, the outcome of WJ-MSCs transplantation was unsuccessful due to multiple factors including oxidative damage. The presence of oxidative stress due to myocardium injury influences fibrous tissue formation, which causes disability of cardiac muscle. Hepatocyte growth factor (HGF), insulin-like growth factor (IGF1), and sonic hedgehog (SHH) are well-known master regulators in antifibrosis when secreted by WJ-MSCs. They showed a beneficial role in the recovery of cardiac fibrosis after WJ-MSCs transplantation. This study hypothesizes whether the reduction of the anti-fibrosis property in WJ-MSCs from oxidative damage can be recovered by overexpression of the HGF, IGF1, or SHH gene. Overexpression was attained by transfection of WJ-MSCs with pCMV3-HGF, pCMV3-IGF1, or pCMV3-SHH followed by $\mathrm{H}_{2} \mathrm{O}_{2}$ exposure and co-culturing with cardiac fibroblasts. Myofibroblast specific markers comprised of alpha-smooth muscle actin ( $\alpha$-SMA) and collagen type 1 (COL1) were evaluated. The WJMSCs treated with $\mathrm{H}_{2} \mathrm{O}_{2}$ influenced the expression of myofibroblastic markers, whereas the overexpression of HGF, IGF1 or SHH reduced myofibroblastic formation. These results indicate that the oxidative stress impaired anti-fibrotic property of WJ-MSCs, leads to an increase of myofibroblasts. Overexpression of anti-fibrotic genes restored the endogenous HGF, IGF1, and SHH alleviating improvement of cardiac function.
\end{abstract}

Keywords: Mesenchymal stem cells, Wharton's jelly, oxidative stress, cardiac fibrosis, antifibrosis

\section{Introduction}

Myocardium infarction (MI) has been reported as a major health problem worldwide. The pathogenesis of MI is from insufficient blood supply to myocardium,

Released online in J-STAGE as advance publication October 25,2019 .

*Address correspondence to:

Dr. Aungkura Supokawej, Department of Clinical Microscopy, Faculty of Medical Technology, Mahidol University, 999 Putthamonthon Sai 4, Putthamonthon, Salaya, Nakhon Pathom 73170, Thailand.

E-mail: aungkura.jer@mahidol.ac.th which leads to apoptosis, limiting proliferative capability of cardiomyocytes and post-MI fibrosis formation (1). Oxidative stress under hypoxic conditions leads to accumulation of reactive oxygen species (ROS), which impacts myocardium function. ROS are toxic cellular radicals, affecting the macrobiological molecules contributing to the induction of cardiomyocyte apoptosis through apoptotic pathways (2), mitochondrial damage (3) as well as inhibition of cardioprotective functions (4).

Nowadays, transplantations are a convincing strategy in myocardium infarction treatment especially mesenchymal stem cells (MSCs) transplantation (5). 
MSCs that are obtained from a perinatal source such as Wharton' jelly derived mesenchymal stem cells (WJMSCs) have been found to have superior biological properties when compared to bone marrow derived MSCs (BM-MSCs) due to high proliferation rates, hypoimmunogenicity (6), lower potential of forming teratomas (7), non-invasive techniques and avoidance of ethical problems (8). Several clinical trial studies showed promising results of treatment, which could be due to the WJ-MSCs properties; immunomodulatory (9), transdifferentiation to cardiomyocytes (10) and releasing cardioprotective paracrines (11) as well as anti-cardiac fibrosis (12). The cardiac fibrosis was initiated during myocardium regeneration and characterized by accumulation of myofibroblasts and high amounts of extracellular matrix (ECM) proteins deposition. These alterations are finally leading to stiffening of the heart and cardiac dysfunction. Unsuccessful findings were frequently found with the poor survival rate and dysfunction of transplanted WJ-MSCs and the underlying cause has not been elucidated. The outcome variances were suggested to be from poor survival of transplanted WJ-MSCs by oxidative stress-induced apoptosis (13). Oxidative stress was a factor reported as a dangerous insult to cell recovery mechanisms underlying pathogenesis and has a negative impact on survival of transplanted WJ-MSCs (14) and fibrosis (15).

Activation of cardioprotective cytokines has been shown during tissue regeneration to promote antifibrosis via induction of metalloproteases (MMP) to degrade ECM, inhibition of the transition from interstitial fibroblasts to myofibroblasts and against proliferation of collagen production of fibroblasts (16). Hepatocyte growth factor (HGF) and insulinlike growth factor (IGF1) have been reported as beneficial agents to prevent apoptosis and fibrosis in cardiovascular diseases (17), liver diseases (18) and pulmonary diseases (19). Sonic hedgehog ( $\mathrm{SHH}$ ) plays a role in the development of the heart blood vessels in the embryonic heart and adult heart (20) and has potential in cardiac repair after myocardial ischemia (21). Therapy of myocardial infarction with WJMSCs showed reduction of fibrosis and promotion of near-normalization of cardiac function (22). WJMSCs released paracrine factors as the signaling mediators of the anti-fibrotic agents. Hepatocyte growth factor and insulin-like growth factor were secreted by WJ-MSCs and have been reported to play a beneficial role in healing of fibrosis (23). Sonic hedgehog is a prototypical morphogen that is involved in cardiovascular regeneration in vivo by inducing the expression of angiogenic cytokines, including vascular endothelial growth factor-1 (VEGF-1), and angiopoietins-1 and -2 (Ang1, Ang2) from interstitial mesenchymal cells (24). Direct injection of SHH has been shown to activate angiogenesis in a critical hindlimb ischemia model (25). The delivery of those genes or proteins reduced fibrosis in numerous in vivo models $(26,27)$.

Here, we investigated the expression level of three candidate anti-fibrotic genes, HGF, IGF1, and SHH in WJ-MSCs during $\mathrm{H}_{2} \mathrm{O}_{2}$-induced reactive oxygen species (ROS), exposure. Then the genetically engineered WJMSCs with each of the anti-fibrotic genes comprised of HGF, IGF1, or SHH pretreated with $\mathrm{H}_{2} \mathrm{O}_{2}$ were cocultured with rat cardiac fibroblasts. We observed the effect of the paracrine fibrogenesis on rat fibroblasts and evaluated for myofibroblastic formation. We used this as a model to observe the effect of those paracrine factors on fibrogenesis and improve their therapeutic effect in vitro. The understanding of these alterations should show great promise for the future use of WJ-MSCs cell therapy in ischemic heart disease (IHD) patients.

\section{Materials and Methods}

\subsection{Isolation and culture of WJ-MSCs}

Umbilical cords were collected from mothers with normal labor as previously described (28). All participants have read and signed the informed consent, which was approved by the Mahidol University Institutional Review Board (protocol no. 147.1311). To isolate Wharton's jelly MSCs (WJ-MSCs), the umbilical cords were cleaned with $70 \%$ ethyl alcohol and washed with $1 \mathrm{X}$ phosphate saline buffer (1X PBS). The umbilical veins and arteries were removed before collection of Wharton's jelly matrix. The tissues were chopped and digested with collagenase type II (Worthington Biochemical Corp., Lakewood, NJ, USA) and $0.25 \%$ trypsin- ethylenediaminetetraacetic acid (EDTA). The cells suspension was harvested and washed twice with PBS. Cells were suspended in complete medium comprised of Dulbecco's Modified Eagle Medium (DMEM) low glucose, 10\% fetal bovine serum (Merck KGaA, Darmstadt, Germany), 1\% Penicillin/Streptomycin, and 1\% GlutaMAX (Gibco, Scientific, Inc., Waltham, MA, USA). The isolated cells were plated on tissue culture dishes and cultured in a $37^{\circ} \mathrm{C}, 5 \% \mathrm{CO}_{2}$ incubator with $95 \%$ humidified air for 24 hours. The supernatant was removed and fresh medium was changed twice a week.

\subsection{Cell and culture of rat cardiac fibroblasts}

Rat cardiac fibroblasts (CF) $(n=5)$ were kindly provided by Dr. Tuempong Wongtawan (Department of Preclinic and Applied Animal Science, Faculty of Veterinary Science, Mahidol University, Thailand). Cells were cultured in DMEM high glucose medium supplemented with $10 \%$ fetal bovine serum under humidified air with $5 \% \mathrm{CO}_{2}$ in a $37^{\circ} \mathrm{C}$ incubator. The medium was changed twice a week. Cells were expanded by trypsinization, 
when cell confluence reached $80 \%$. Confluent cells were washed twice with $1 \mathrm{X}$ PBS and incubated with $0.25 \%$ trypsin-EDTA at $37^{\circ} \mathrm{C}$ for 5 minutes. Afterward, cell suspension was harvested by centrifugation at $1,500 \mathrm{rpm}, 25^{\circ} \mathrm{C}$ for 5 minutes, and re-suspended with appropriate concentrations in culture medium.

\subsection{WJ-MSCs characterization}

WJ-MSCs in all experiments were performed as previously described (28). WJ-MSCs were characterized according to the minimal criteria for defining MSCs, stated by the International Society for Cellular Therapy (29). MSCs immunophenotype and mesodermal differentiation potential were performed. Briefly, cell surface markers expression of MSCs were evaluated from WJ-MSCs passage $3^{\text {rd }}-5^{\text {th }}$. Cells were collected and incubated with antibodies specific for CD105, CD73, CD90, CD34 and CD45 (BD Pharmingen ${ }^{\mathrm{TM}}$, San Jose, CA, USA) followed by examination with a BD FACSCantoTM II Flow Cytometer and analysis by FACSDIVA Software version 6.1.3 (BD Biosciences, San Jose, CA, USA).

For mesodermal differentiation properties of MSCs, WJ-MSCs were cultured with osteogenic and adipogenic differentiation medium (Stem Cell Technologies, Vancouver, Canada) as previously described (28). WJMSCs were cultured with differentiation medium for 35 days. The differentiated cells were stained with Alizarin Red S and Oil Red $\mathrm{O}$ to evaluate for mineralized calcium deposition and fat deposition, respectively. The cytochemically stained cells were observed using a microscope and photographed.

\subsection{Cell viability assay}

MTT assay was employed to determine percent cell viability of WJ-MSCs during $\mathrm{H}_{2} \mathrm{O}_{2}$ treatment. WJMSCs were cultured with $1 \times 10^{3}$ seeding density for each well of a 96-well plate for 24 hours, followed by culture with growth medium containing 200, 500 and $1,000 \mu \mathrm{M} \mathrm{H}_{2} \mathrm{O}_{2}$. Cell viability of treated and control cells were analyzed as described. First, samples were washed with the DMEM twice. $50 \mu \mathrm{L}$ of MTT (3-(4,5-dimethylthiazol-2-yl)-2,5-diphenyltetrazolium bromide) reagent $(1 \mathrm{mg} / \mathrm{mL})$ was added to the sample and incubated at $37 \mathrm{o} \mathrm{C}$, in a $5 \% \mathrm{CO}_{2}$ incubator for 4 hours. Then, the supernatant was removed, $100 \mu \mathrm{L}$ of dimethyl sulfoxide was added, and the plate was shaken for $15 \mathrm{~min}$ to completely dissolve the formazan crystals before measuring the absorbance at $570 \mathrm{~nm}$. Cell viability was calculated using the following formula.

$$
\% \text { Cell viability }=
$$

$\left(\frac{\text { Absorbance of sample-Absorbance of blank }}{\text { Absorbance of control-Absorbance of blank }}\right) \times 100$

\subsection{Quantitative reverse transcriptase-polymerase chain reaction ( $q R T-P C R$ )}

The experimental samples from WJ-MSCs and $\mathrm{CF}$ were collected in TRIzol ${ }^{\circledR}$ reagent (Invitrogen; Thermo Fisher Scientific, Inc., Waltham, MA, USA) and RNA isolation was performed using Direct-zol columns (Zymo Research, Irvine, CA, USA). The concentration of RNA was determined with a Nanodrop 2000 spectrophotometer (Thermo Fisher Scientific, Inc.). Next, First-strand cDNA was synthesized with the isolated RNA ( $1 \mu \mathrm{g} /$ sample) using a cDNA Synthesis Kit, (Biotechrabbit, Berlin Germany). The expression of anti-fibrotic and myofibroblastic genes were investigated by qPCR using KAPA SYBR ${ }^{\circledR}$ Fast qPCR Kit (KAPABIOSYSTEMS, Massachusetts USA). The quantitative PCR was run with a CFX96 (Bio-Rad Laboratories, Inc.). The conditions of qPCR were set as follows: denaturation; $95^{\circ} \mathrm{C}$ for 3 minutes; followed by 40 cycles of $95^{\circ} \mathrm{C}$ for 3 seconds, annealing; $60^{\circ} \mathrm{C}$ for 30 seconds and elongation; $72^{\circ} \mathrm{C}$ for 45 seconds. The primer sequences were designed as follows: HGF forward, 5'-GGGCTGAAAAGATTGGATCA-3' and reverse, 5'-TAATTTTGTGTATCCATTTTGCAT-3'; SHH forward, 5'-GTAAGGACAAGTTGAACGCTTTG-3', and reverse, 5'-ATATGTGCCTTGGACTCGTAGTA -3'; and GAPDH forward, 5'-CAACTACATGGTTTAC ATGTTCCAA-3' and reverse, 5'-CAGCCTTCTCCAT GGTGGT-3'. The primers for CF were as follows: rCOL1 forward, 5'-AGGCATAAAGGGTCATCGTG-3' and reverse, 5'-ACCGTTGAGTCCATCTTTGC-3'; rSMA forward, 5'-ACTGGGACGACATGGAAAAG-3', and reverse, 5'-TACATGGCAGGGACATTGAA-3'; rGA PDH forward, 5'-AGCTCATTTCCTGGTATGACAA -3' and reverse, 5'-GGTATTCGAGAGAAGGGAGGG -3 '. The expression of glyceraldehyde-3-phosphate dehydrogenase (GAPDH) were also quantified and applied as internal control in parallel with the target genes. The expression level of each gene was calculated by the $\Delta \Delta \mathrm{Ct}$ method and presented in fold changes using Bio-Rad CFX Manager version 3.1 (Bio-Rad Laboratories, Inc.) (30).

\subsection{Immunofluorescence staining}

Cells were seeded and cultured on coverslips. The coverslips of cells were washed with 1X PBS followed by fixation with $4 \%$ paraformaldehyde for 20 minutes and washing with $1 \mathrm{X}$ PBS. Permeabilization was carried out using $0.3 \%$ Triton $^{\mathrm{TM}} \mathrm{X}-100$ (Merck KGaA) for 5 minutes and non-specific reaction blocking was performed with $3 \%$ bovine serum albumin (BSA) (Invitrogen; Thermo Fisher Scientific, Inc.) in PBS for 1 hour. Samples were incubated with anti-HGF (Santa Cruz, CA USA), anti-IGF1 (Abcam, NY USA) and anti-SHH (Abcam, NY USA) at $4^{\circ} \mathrm{C}$ overnight. 
Samples were then washed twice with PBS to remove the excess primary antibodies, followed by incubation with Alexa flour 488 conjugated secondary antibody at room temperature for 1 hour. Cells were washed with PBS and mounted with Antifade Mounting with DAPI solution (Invitrogen; Thermo Fisher Scientific, Inc.,). The observation was performed using a confocal laser scanning microscope, the fluorescent micrographs were captured and analyzed with FluoView FV1000 Software version 3.01 (Olympus Corp., Tokyo, Japan).

\subsection{Western blot analysis}

HGF, IGF1, and SHH were quantified by collection of the condition medium and centrifugation at 1,500 rpm for 5 minutes to discard cell residue (31). Protein concentration was determined by using Bradford assay (Bio-Rad, CA, USA). The samples were mixed with dye and boiled for 5 minutes to denature the proteins. Samples were loaded in 10\% SDS-PAGE and transferred to nitrocellulose membrane (BioRad, CA, USA). Blocking of non-specific antibody binding was performed by incubating with $5 \%$ nonfat dried milk in TBST buffer $(0.1 \mathrm{M}$ Tris- $\mathrm{HCl}$ and $0.1 \%$ Tween-20, $\mathrm{pH}=7.5$ ) for 1 hour. Membranes were submerged with each antibody as follows; rabbit antihuman HGF polyclonal (Santa Cruz, CA, USA), mouse anti-SHH monoclonal (Abcam, NY, USA) and mouse anti IGF1 monoclonal (Abcam, NY USA). Next, the membranes were incubated with secondary antibody conjugated with horseradish peroxidase (Cell Signaling Technology, MA, USA). The signal of labelled proteins was done using the ECLTM prime Western blotting detection reagent (Amersham, UK). Images were scanned with the ChemiDoc ${ }^{\mathrm{TM}}$ MP Imaging System (Bio-Rad, UK).

\subsection{Transfection}

All plasmids, including pCMV3-HGF, pCMV3-IGF1 and pCMV3-SHH were purchased from Sino Biological Inc., China. The plasmids were transformed into Top10 competent E. coli and plated on LB agar plates supplemented with ampicillin. The bacterial colonies were picked and plated into LB medium supplemented with ampicillin. The bacteria were inoculated in shaking culture at $37^{\circ} \mathrm{C}$ for 18 hours. Plasmid DNA was extracted with a FavorPrep ${ }^{\mathrm{TM}}$ Plasmid DNA extraction mini kit (Favogen Biotech Corp, Taiwan). $\mathrm{PCR}$ and restriction enzyme digestion (XbaI and KpnI) were applied to confirm the correct clone from each plasmid. The correct clones were propagated to obtain a large volume. The desired plasmids were extracted by Geneaid ${ }^{\text {TM }}$ Midi Plasmid kit (Geneaid, NY USA) for transfection into WJ-MSCs. WJ-MSCs were cultured in 100-mm dishes at a density of $2 \times 10^{6}$ cells. Next, cells were transfected with $24 \mu \mathrm{g}$ of plasmid using Lipofectaminne 2000 (Invitrogen, CA USA) according to the manufacturer's protocol (32). Briefly, $24 \mu \mathrm{g}$ of plasmid were mixed with Opti-MEM to a final volume of $900 \mu \mathrm{l}$ and incubated for 5 minutes at room temperature. The mixture of diluted plasmids and the Lipofectamine $^{\mathrm{TM}} 2000$ in Opti-MEM was prepared and incubated for 20 minutes at room temperature to generate the DNA-Lipofectamine 2000 complexes. This mixture was applied to cell culture plates and filled with medium without antibiotic.

\subsection{Amplification of plasmid by polymerase chain reaction $(P C R)$}

The DNA template for the pCMV3-HGF, pCMV3IGF1 and pCMV3-SHH containing CDS of interest, was amplified using PCR. The primers of pCMV3 (forward, 5'-CAGGTGTCCACTCCCAGGTCCAAG-3' and reverse, 5'-GGCAACTAGAAGGCACAGTC GAGG-3'); were performed for PCR reaction using I-Taq plus DNA polymerase (iNtRON Biotechnology, Inc., Korea) according to manufacturer's instructions as described. The PCR reaction was performed using the following cycling protocol: initial activation step at $94^{\circ} \mathrm{C}$ for 2 minutes, followed by 30 cycles of denaturation at $94^{\circ} \mathrm{C}$ for 20 seconds, annealing at $62^{\circ} \mathrm{C}$ for 10 seconds, extension at $72^{\circ} \mathrm{C}$ for 1.30 minutes and final extension at $72^{\circ} \mathrm{C}$ for 7 minutes.

\subsection{Statistical analysis}

All data of the study were collected from 6 donors, the experiments were performed independently and at least three samples were applied for each experiment. The data is presented as mean \pm standard error of the mean. To test statistical difference of mean, Mann-Whitney $U$ test was applied and $P<0.05$ was considered significant difference. Statistical tests were done using PASW software version 18 (IBM Corp., Armonk, NY, USA).

\section{Results}

\subsection{WJ-MSC characterization}

WJ-MSCs over the first 5-6 days of culture displayed fibroblast-like morphology (Figure 1A). The specific MSC markers (CD90, CD73 and CD105) and hematopoietic cells markers (CD34 and CD45) were analyzed with flow cytometry (Figure 1B). The positive expressions were present on WJ-MSCs; CD90, CD73, CD105. WJ-MSCs were mostly negative for HSC markers; CD34, CD45. To explore the differentiation potential towards osteoblasts and adipocytes, WJ-MSCs were cultured with osteogenic and adipogenic differentiation medium followed by cytochemical staining with Alizarin Red S and Oil Red 
A

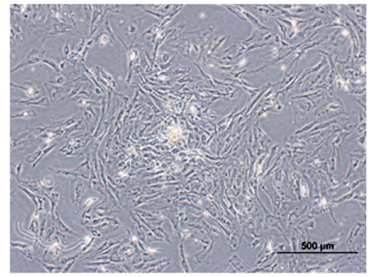

B

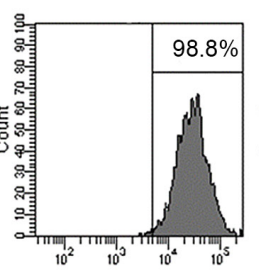

CD90 APC-A

C

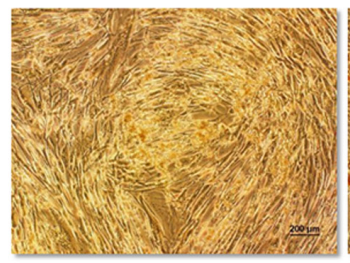

control

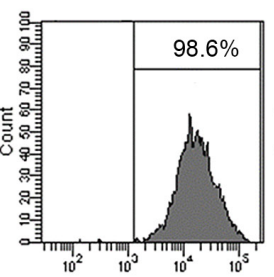

CD73 PE-Cy7-A

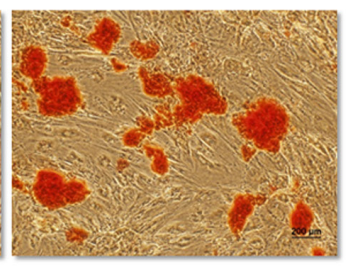

Osteogenic differentiation

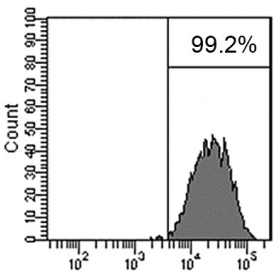

CD105 PE-A
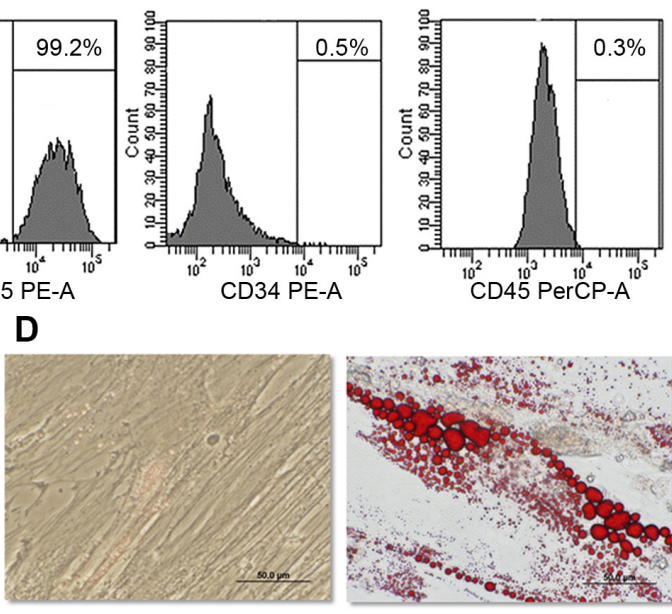

control

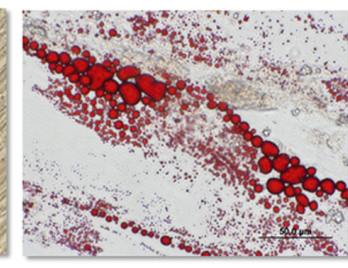
differentiation
Adipogenic

Figure 1. The characterization of WJ-MSCs. (A) The spindle-shaped morphology of WJ-MSCs was observed. (B) Flow cytometric analysis of WJ-MSCs are more than $90 \%$ positive for MSC markers, CD105, CD90, CD73 and less than 2\% positive for hematopoietic markers, CD34, CD45. For the multilineage differentiation study, WJ-MSCs were cultured with osteogenic and adipogenic differentiation medium followed by cytochemical staining, (C) Alizarin red S and (D) Oil red O, respectively. WJ-MSCs exhibited positive staining under both conditions.

O, respectively. The differentiated cells were positive for both cytochemical stains which indicated cell differentiation toward osteoblast-like (Figure 1C) and adipocyte-like cells (Figure 1D).

\subsection{Cell viability assay}

The effect of $\mathrm{H}_{2} \mathrm{O}_{2}$ on the viability of WJ-MSCs was investigated. Cells were treated with various concentration of $\mathrm{H}_{2} \mathrm{O}_{2}$ for 24 and 48 hours before assessing cell viability using MTT assay. The viability of WJ-MSCs after $\mathrm{H}_{2} \mathrm{O}_{2}$ treatment was expressed as a percentage relative to control (Figure 2A). Results showed that the viability of WJ-MSCs was decreased in a dose- and time-dependent manner after $\mathrm{H}_{2} \mathrm{O}_{2}$ treatment. At $1,000 \mu \mathrm{M}$ of $\mathrm{H}_{2} \mathrm{O}_{2}$ treatment, the viability of WJ-MSCs was significantly decreased compared with control. Microscopic examination of the cells after $\mathrm{H}_{2} \mathrm{O}_{2}$ treatment for 7 days showed low cell density in $1,000 \mu \mathrm{M} \mathrm{H}_{2} \mathrm{O}_{2}$ treatment compared with control (Figure 2B).

\subsection{Anti-fibrotic gene and protein expression in $W J$ - MSCs under ROS condition}

To determine the expression level of HGF, IGF1, and SHH in WJ-MSCs, real-time PCR and immunofluorescence staining were performed. WJ-
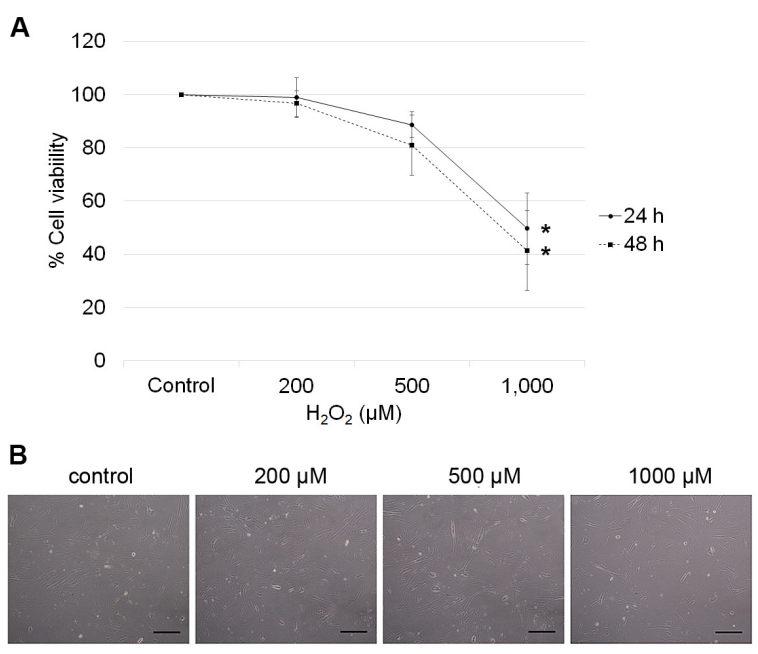

Figure 2. The viability of WJ-MSCs after $\mathrm{H} 2 \mathrm{O} 2$ treatment. (A) WJ-MSCs were treated with $\mathrm{H}_{2} \mathrm{O}_{2}$ at 200, 500 and 1,000 $\mu \mathrm{M}$ for 24 and 48 hours. The viability of the cells was assessed by MTT assay and presented as a mean percentage relative to the control \pm SEM of six separate experiments. The viability of WJ-MSCs was reduced by $\mathrm{H}_{2} \mathrm{O}_{2}$ treatment in a dose- and time-dependent manner. ${ }^{*} p<0.05 v s$. control group. (B) Microscopic examination of WJ-MSCs after $\mathrm{H}_{2} \mathrm{O}_{2}$ treatment for 7 days revealed a low cellular density in $\mathrm{H}_{2} \mathrm{O}_{2}$ treated cells compared with control group. Scale bar, $200 \mu \mathrm{m}$.

MSCs were treated with 200, 500, and $1,000 \mu \mathrm{M} \mathrm{H}_{2} \mathrm{O}_{2}$ for 24 hours before harvesting mRNA or staining with anti-fibrotic markers. In comparison with control group, 
the expression levels of HGF, IGF1, and SHH were significantly reduced after $\mathrm{H}_{2} \mathrm{O}_{2}$ treatment in a dosedependent manner (Figure 3A). Immunofluorescence staining of HGF, IGF1, and SHH showed decreased fluorescence intensity of the three markers in $\mathrm{H}_{2} \mathrm{O}_{2}$ treated cells compared with control (Figure 3B).

\subsection{Myofibroblastic gene expression in cardiac} fibroblasts $(\mathrm{CF})$ after co-culture with $\mathrm{H}_{2} \mathrm{O}_{2}$-treated WJMSCs

To determine the effect of $\mathrm{H}_{2} \mathrm{O}_{2}$ on the anti-fibrotic properties of WJ-MSCs, WJ-MSCs were plated in transwells and cultured with medium supplemented with 200, 500, and $1,000 \mu \mathrm{M} \mathrm{H}_{2} \mathrm{O}_{2}$ for 24 hours. The $\mathrm{H}_{2} \mathrm{O}_{2}$-treated WJ-MSCs were then co-cultured with CF for 48 hours. Myofibroblastic specific markers; alpha-smooth muscle actin (SMA) and collagen type 1 (COL1) were investigated in CF. The gene expression of COL1 and SMA in CF were not significantly different compared with control (Figure 4A), whereas the immunofluorescent micrographs showed the highest fluorescent intensity for COL1 and SMA signal in the

A

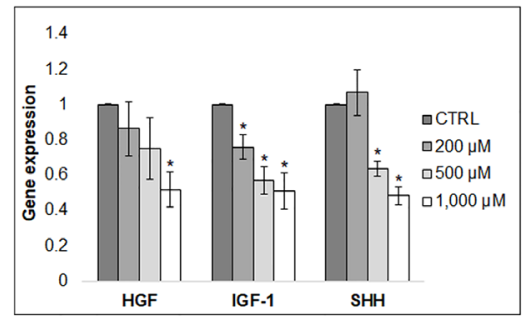

B
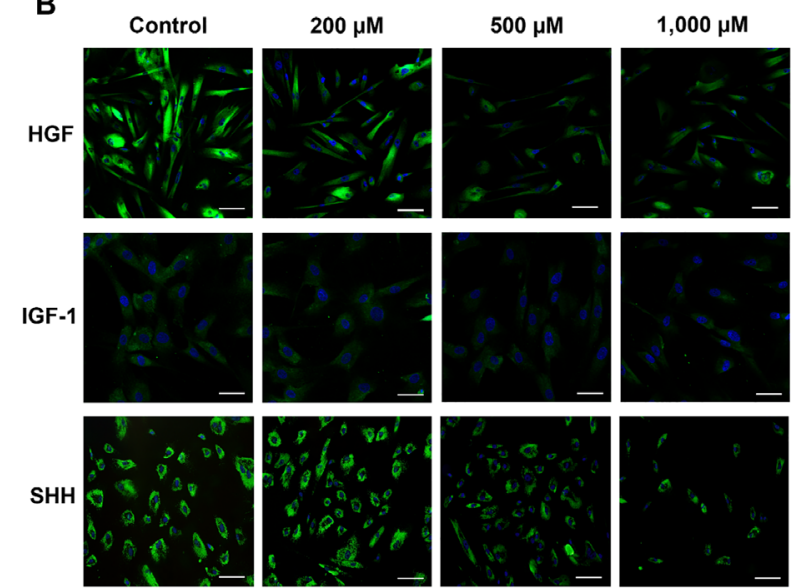

Figure 3. Anti-fibrotic factors expression in WJ-MSCs after $\mathbf{H}_{2} \mathrm{O}_{2}$ treatment. (A) The levels of HGF, IGF1, and SHH genes were significantly reduced in $\mathrm{H}_{2} \mathrm{O}_{2}$-treated WJ-MSCs when compared with control group. Data were expressed as mean \pm SEM $(n=6),{ }^{*} p<0.05 v s$. control. (B) Immunofluorescent micrographs of HGF, IGF1, and SHH in WJ-MSCs after treatment with various concentrations of $\mathrm{H}_{2} \mathrm{O}_{2}$. Low fluorescent intensity of HGF and SHH was obviously observed in 500 and $1,000 \mu \mathrm{M} \mathrm{H}_{2} \mathrm{O}_{2}$-treated cells compared with control, whereas the fluorescence intensity of IGF-1 was similar to control. Scale bars, $100 \mu \mathrm{m}$. WJ-MSCs, Wharton's jelly-derived WJ-MSCs; HGF, hepatic growth factor; IGF1, insulin-like growth factor; $\mathrm{SHH}$, sonic hedgehog.
$1,000 \mu \mathrm{M} \mathrm{H}_{2} \mathrm{O}_{2}$ treatment group indicating an increased myofibroblastic phenotype in CF (Figure 4B). Thus, we decided to use $1,000 \mu \mathrm{M} \mathrm{H}_{2} \mathrm{O}_{2}$ treated WJ-MSCs for the subsequent experiments.

3.5. Anti-fibrosis gene and protein expression of WJMSCs after transfection

In order to improve anti-fibrotic properties of WJMSCs, the WJ-MSCs were transfected with pCMV3HGF, pCMV3-IGF1, or pCMV3-SHH for 48 hours using Lipofectamine ${ }^{\mathrm{TM}}$ 2000. The timeline of the

A

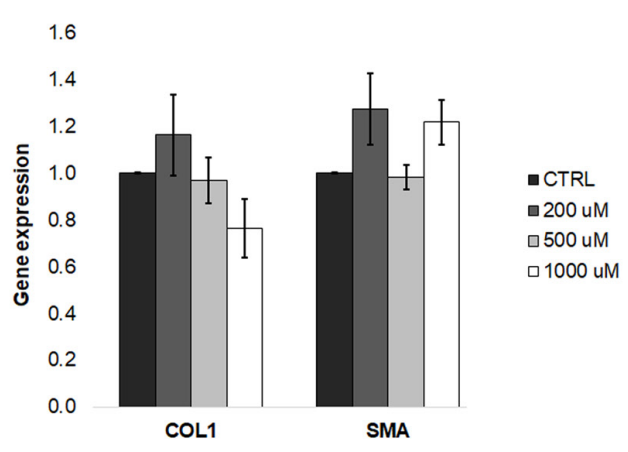

$\mathbf{B}$ COL1

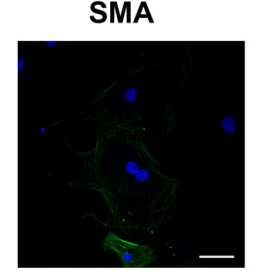

CTRL
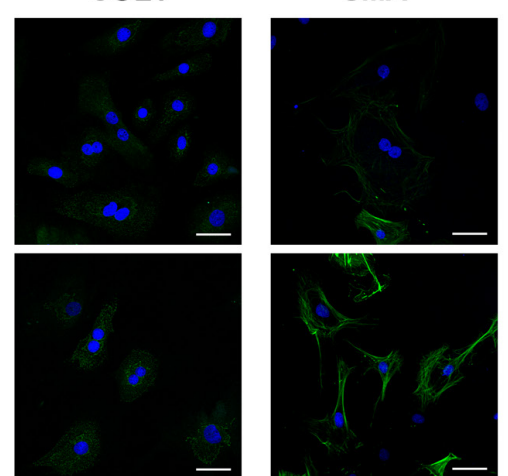

$200 \mu M$
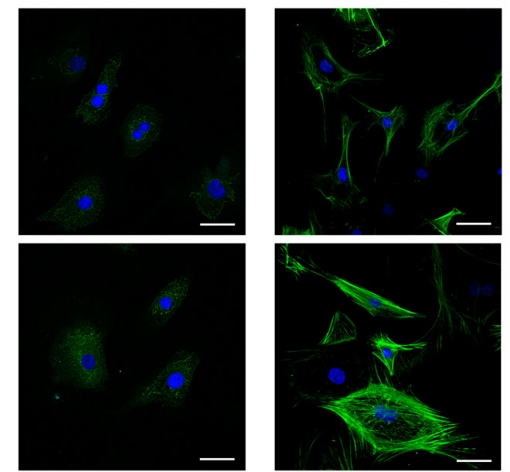

$1,000 \mu \mathrm{M}$
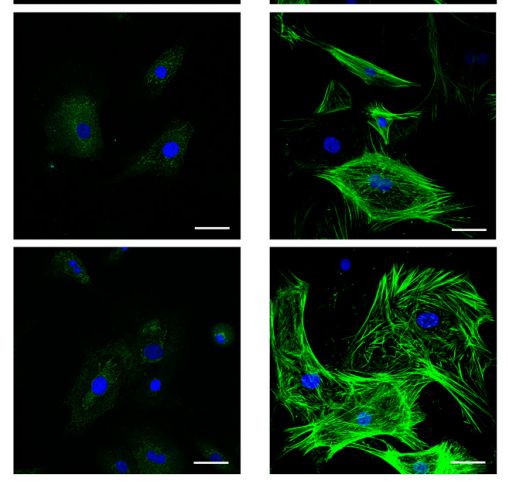

Figure 4. Myofibroblastic gene expression in $\mathrm{CF}$ after coculture with $\mathrm{H}_{2} \mathrm{O}_{2}$-treated WJ-MSCs. (A) Gene expression of COL 1 and SMA in $\mathrm{H}_{2} \mathrm{O}_{2}$-treated WJ-MSCs were not significantly different when compared with control. Data are expressed as mean $\pm \operatorname{SEM}(n=3)$. (B) Immunofluorescence micrographs showed the increased fluorescent intensity of COL1 and SMA in CF after co-culture with $\mathrm{H}_{2} \mathrm{O}_{2}$-treated WJMSCs, in particular at $1,000 \mu \mathrm{M}$ of $\mathrm{H}_{2} \mathrm{O}_{2}$. Scale bars, $100 \mu \mathrm{m}$. COL1, collagen type I; SMA, smooth muscle actin. 
A

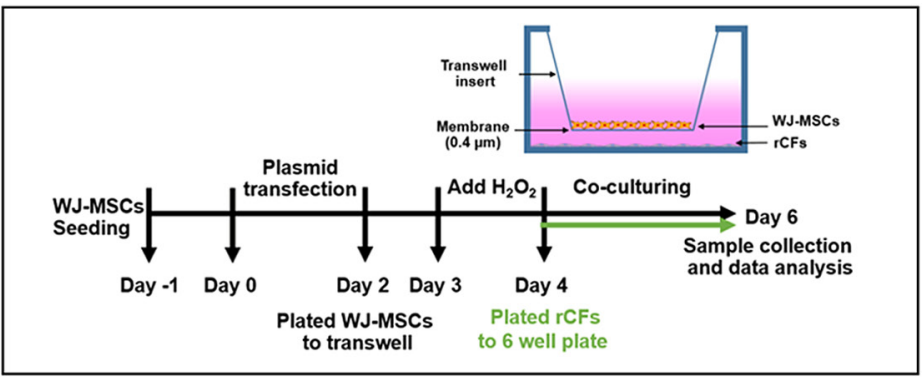

B

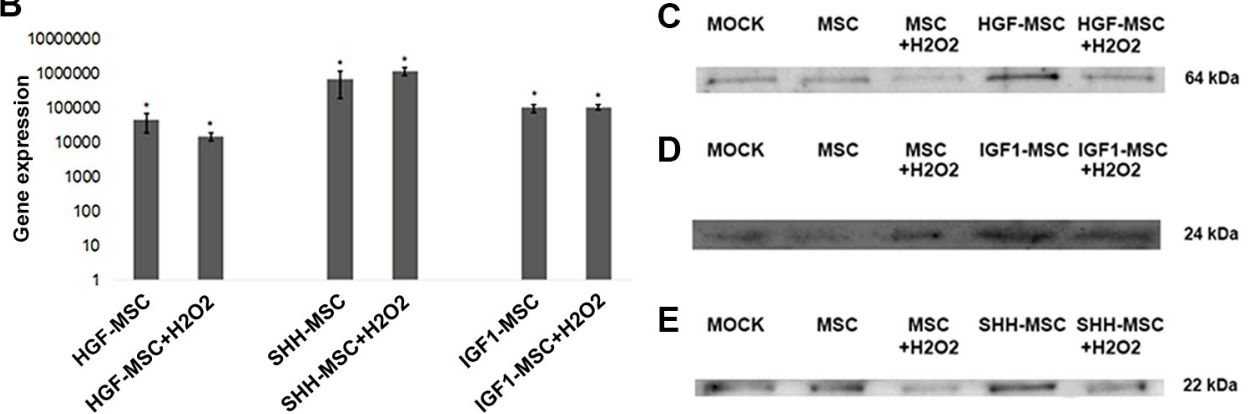

Figure 5. Overexpression of anti-fibrosis factors in WJ-MSCs. (A) Schematic diagram represents the process of WJ-MSC transfection with anti-fibrosis genes. (B) Analysis of HGF, IGF1 and SHH mRNA expression in transfected WJ-MSCs at day 6. The levels of HGF, SHH and IGF1 were significantly up-regulated in both transfected MSCs and transfected $\mathrm{MSCs}_{\text {after }} \mathrm{H}_{2} \mathrm{O}_{2}$ treatment. Data are expressed as mean $\pm \operatorname{SEM}(n=3),{ }^{*} p<0.05 v s$. control. Western blot analysis of (C) HGF, (D) IGF1, (E) SHH in conditioned medium of WJ-MSCs are shown. Level of HGF, IGF1, and SHH in conditioned medium from transfected MSCs were higher than those in MSCs. These proteins were decreased after $\mathrm{H}_{2} \mathrm{O}_{2}$ treatment.

process is shown in Figure 5A. RNA and conditioned medium were collected from transfected MSCs and expression of HGF, IGF1, and SHH were investigated before co-culture with CF. The expression of HGF, SHH, and IGF1 genes in transfected WJ-MSCs were approximately up to 10,000 fold higher than control and were not reduced after $\mathrm{H}_{2} \mathrm{O}_{2}$ treatment (Figure 5B). The examination of the protein expression in conditioned media performed by Western blot analysis showed decreased HGF, IGF1, and SHH proteins in conditioned media of $\mathrm{H}_{2} \mathrm{O}_{2}$-treated WJ-MSCs. These proteins were increased in conditioned media of transfected WJ-MSCs (HGF-, IGF1-, SHH-MSC) and decreased after $\mathrm{H}_{2} \mathrm{O}_{2}$ treatment. However, compared to non-transfected WJMSCs, the HGF, IGF1, and SHH protein concentrations tended to be higher in transfected MSCs after $\mathrm{H}_{2} \mathrm{O}_{2}$ treatment (Figure 5C-E).

\subsection{Myofibroblastic gene and protein expression of $C F$ after co-culture with transfected WJ-MSCs}

To determine whether the expression of anti-fibrotic secreted factors from transfected WJ-MSCs could affect myofibroblast properties in $\mathrm{CF}$, we investigated the myofibroblastic markers; COL1 and SMA in $\mathrm{CF}$ after co-culture with WJ-MSCs for 48 hours (Figure 5A). The conditions of WJ-MSCs for coculture study were as follows; no MSC, -MOCK, $-\mathrm{MSC},-\mathrm{MSC}+\mathrm{H}_{2} \mathrm{O}_{2},-\mathrm{MSC}+\mathrm{pCMV} 3(\mathrm{HGF} / \mathrm{IGF} 1 /$ $\mathrm{SHH}),-\mathrm{MSC}+\mathrm{pCMV} 3(\mathrm{HGF} / \mathrm{IGF} 1 / \mathrm{SHH})+\mathrm{H}_{2} \mathrm{O}_{2}$.
As determined by immunofluorescence staining, the fluorescent intensity of COL1 and SMA in CF were lower after co-culture with MSC and MSC + pCMV3 compared with CF (Figure 6-8). The fluorescent signal tended to be increased after co-culture with $\mathrm{H}_{2} \mathrm{O}_{2}$ treated MSCs compared with MSCs, however, the expression of these myofibroblast markers tended to be decreased after co-culture with $\mathrm{MSC}+\mathrm{pCMV} 3+\mathrm{H}_{2} \mathrm{O}_{2}$ (Figure 6A,7A, 8A). For the gene expression study, SMA expression was found to have the same trend as the results of fluorescent intensity (Figure 6B, 7B, 8B). The expression of COL1 from all conditions was not much different among the co-culture conditions (Figure $6 \mathrm{~B}, 7 \mathrm{~B}, 8 \mathrm{~B})$. All evidence showed the efficacy of antifibrotic factors overexpressed-MSC diminished the transformation from fibroblasts to myofibroblasts.

\section{Discussion}

Stem cell therapy has become a potential novelty for treating degenerative diseases, in particular, cell transplantation in ischemic heart disease (IHD). WJMSCs are considered to be a promising stem cell type for treating IHD due to their high number in the tissue, which can easily be isolated by low-invasive methods (33). Previous research reported the potential of WJ-MSCs to differentiate into cardiomyocytes, neovascularization and cardioprotective effects via paracrine factors (34). In the present study, WJMSCs exhibited typical MSC characteristics including 

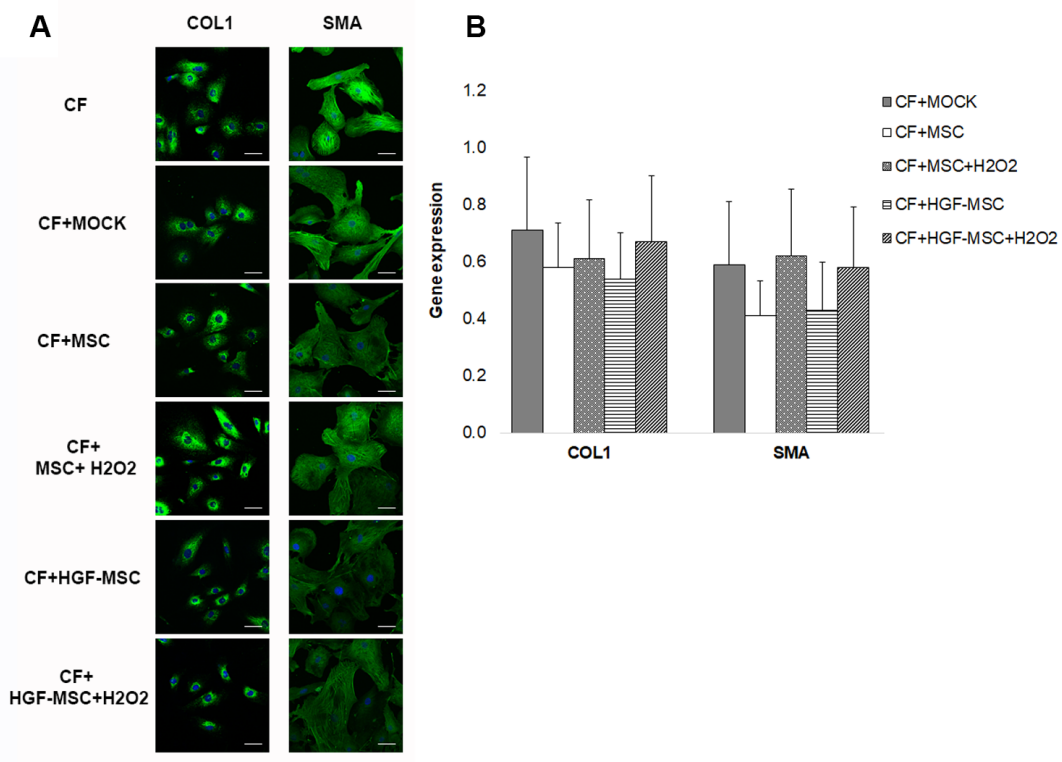

Figure 6. Expression of myofibroblast markers in $\mathbf{C F}$ after co-culture with or without HGF-overexpressed WJ-MSCs. (A) Immunofluorescence micrographs of CF taken at the 48 hours after co-culture with WJ-MSCs. A low fluorescent intensity of COL1 and SMA was observed in $\mathrm{CF}+\mathrm{MSC}$ and CF+HGF-MSC. Scale bar, $50 \mu \mathrm{m}$. (B) The expression of myofibroblastic genes was assessed in $\mathrm{CF}$ at 48 hours after co-culture. The expression of COL1 and SMA gene was concordant with the immunofluorescence staining. Data are expressed as mean $\pm \mathrm{SEM}$ $(n=3)$.
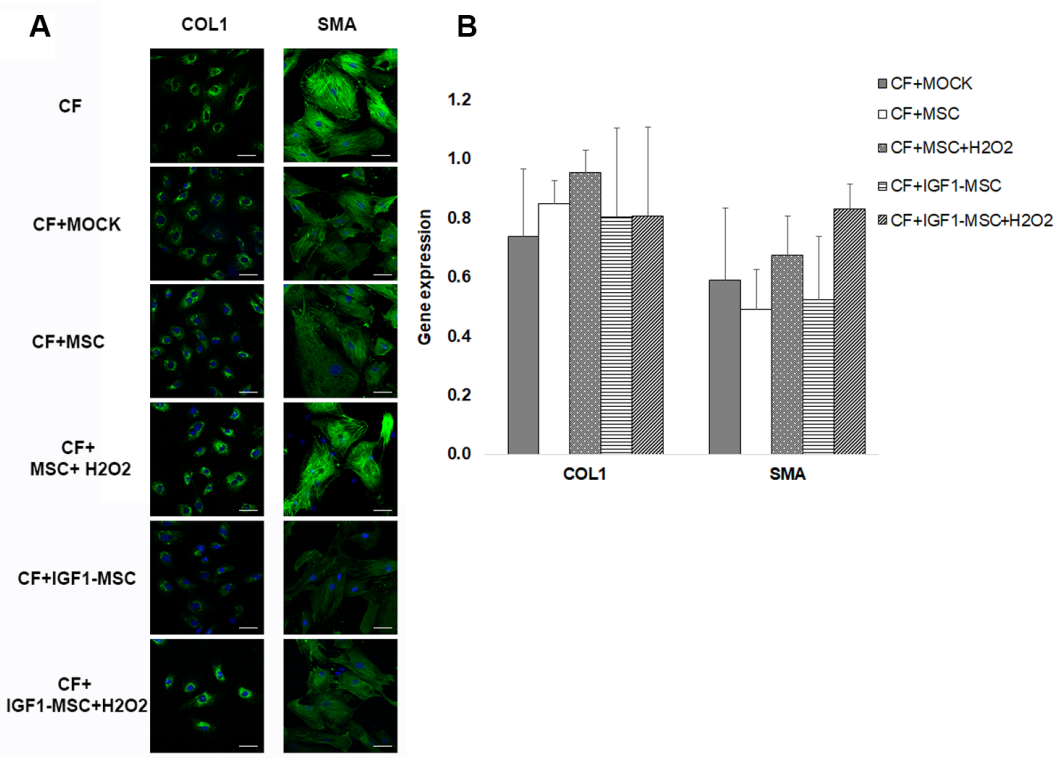

Figure 7. Expression of myofibroblast markers in $\mathrm{CF}$ after co-culture with or without IGF1-overexpressed WJ-MSCs.

(A) Immunofluorescent micrographs of CF taken at the 48 hours after co-culture with WJ-MSCs. A low fluorescent intensity of COL1 and SMA was observed in CF+MSC and CF+IGF1-MSC. Scale bar, $50 \mu \mathrm{m}$. (B) The myofibroblastic gene level was assessed in CF 48 hours after co-culture. The expression of COL1 and SMA gene was concordant with the immunofluorescence staining. Data are expressed as mean \pm SEM $(n=3)$.

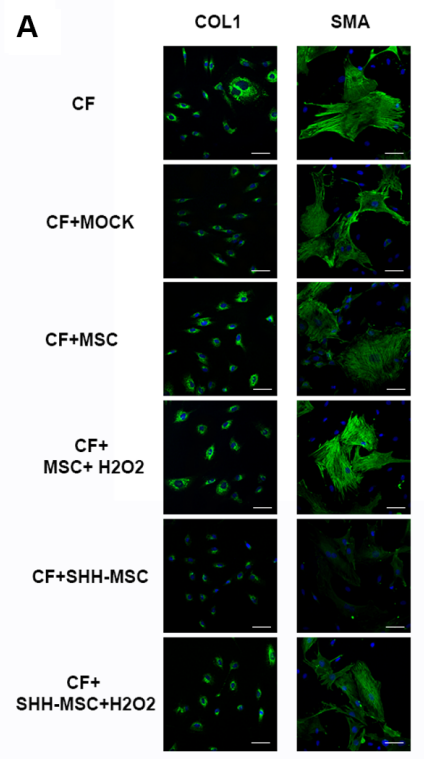

B

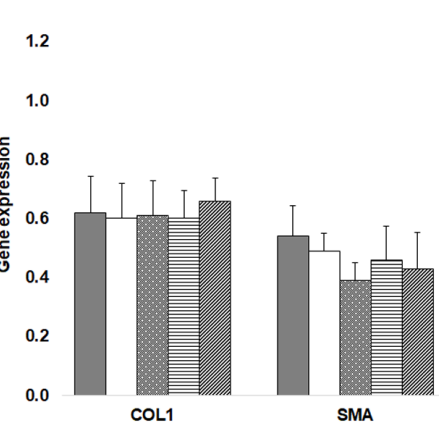

$\square \mathrm{CF}+\mathrm{MOCK}$

$\square C F+M S C$

m $\mathrm{CF}+\mathrm{MSC}+\mathrm{H} 2 \mathrm{O} 2$

ECF+SHH-MSC

2. $\mathrm{CF}+\mathrm{SHH}-\mathrm{MSC}+\mathrm{H} 2 \mathrm{O} 2$

Figure 8. Expression of myofibroblast markers in $\mathrm{CF}$ after co-culture with or without SHH-overexpressed WJ-MSCs. (A) Immunofluorescent micrographs of $\mathrm{CF}$ taken at the 48 hours after co-culture with WJ-MSCs. A low fluorescent intensity of COL1 and SMA was observed in CF+MSC and CF +SHH-MSC. Scale bar, $50 \mu \mathrm{m}$. (B) The myofibroblastic gene expression was assessed in CF 48 hours after co-culture. The expression of COL1 and SMA gene was not different among tested groups. Data are expressed as mean $\pm \operatorname{SEM}(n=3)$. 
expression of cell surface markers (CD90, CD73 and CD105) and differentiation potential into mesodermal lineages (osteoblasts and adipocytes). It had been shown that the response of MSCs to oxidative insult was different according to the origin of MSCs (35). Therefore, the selection of MSCs used for transplantation must be a concern and appropriate for the specific use. Even though MSCs from different origins have similar characteristics, the type of MSCs must be carefully considered to enhance the outcome of cell transplantation.

WJ-MSCs have been shown to exert anti-fibrotic properties although the underlying mechanism is poorly characterized. WJ-MSCs are able to secrete many anti-fibrotic cytokines including HGF, fibroblast growth factor 2 (FGF2), connective tissue growth factor (CTGF), Tumor necrosis factor-inducible gene 6 (TSG6), IGF1 and SHH, which could account for their therapeutic effects (36). Recently, WJ-MSCs treatment for fibrotic diseases has reached clinical trial phase 2, especially in liver fibrosis and cardiac fibrosis. Post myocardial infarction usually develops cardiac fibrosis, which impairs cardiac function. Therefore, the reduction of cardiac fibrosis might be a useful strategy for treatment of heart failure. Even though, it has been demonstrated that administration of WJ-MSCs in a myocardial infarction model decreased cardiac fibrosis (37) but unsuccessful findings were frequently found with poor survival rate and dysfunction of transplanted WJ-MSCs.

Oxidative stress was reported to be an important factor influencing the transplanted WJ-MSCs survival and function. Here, we focused on the role of oxidative stress in anti-fibrotic properties of WJ-MSCs. We hypothesized that oxidative stress may diminished the anti-fibrotic property of WJ-MSCs whereas the overexpression of HGF, IGF1 or SHH genes can reconcile this function. Our results demonstrated that the gene and protein levels of anti-fibrosis factors such as HGF, IGF1 and SHH were significantly decreased after exposure to $\mathrm{H}_{2} \mathrm{O}_{2}$ in a dose dependent manner. HGF, IGF1 and SHH are novel anti-fibrotic cytokines. It has been shown that HGF prevented the progression of fibrogenesis in multiple organs such as lung, liver and heart $(36,38,39)$. IGF1 exerts the beneficial effect on cardiac repair by enhancing angiogenesis and improving myocardial function (40). Previous studies demonstrated that $\mathrm{SHH}$ inhibited cardiac fibrosis, at least in part, by stimulating the production of paracrine factors that exert anti-fibrotic action (41). SHH gene therapy also enhanced neovascularization in rat myocardial infarction through the upregulation of angiogenic-associated genes including VEGF, stromal cell-derived factor 1 (SDF-1), angiopoeitin, and IGF1 (42). Reactive oxygen species have been shown to activate latent transforming growth factor beta (TGF- $\beta$ ) and induce the expression of profibrogenic cytokines such as TGF- $\beta$ and angiotensin II (ANG) (43), which are negative regulators of HGF (44). It is possible that $\mathrm{H}_{2} \mathrm{O}_{2}$ reduced the expression of anti-fibrosis genes and proteins in WJ-MSCs by stimulating the expression of its antagonist, however, the underlying mechanism was largely unknown. The consequence of downregulation of HGF, IGF1 and SHH under oxidative stress conditions might impair anti-fibrosis properties of WJ-MSCs. Therefore, the anti-fibrosis potential of WJ-MSCs was further investigated by coculturing with rat cardiac fibroblasts (CF). The results showed that the expression of myofibroblastic specific markers; COL1 and SMA in CF was increased after co-culture with $\mathrm{H}_{2} \mathrm{O}_{2}$ treated WJ-MSCs. This finding might affect to the activation of interleukin-6 (IL-6) through phosphatidylinositol 3-kinase (PI3K), protein kinase $\mathrm{B}(\mathrm{Akt})$, and glycogen synthase kinase 3 beta (GSK3 $\beta$ ) downstream molecules (45). The upregulation of IL- 6 plays a key role in the production of TGF- $\beta$ via the JAK/STAT3 signaling pathway, which enhances the transdifferentiation of fibroblasts to myofibroblasts (46). Therefore, oxidative stress might be one important mechanism that attenuates the anti-fibrotic ability of WJ-MSCs. ROS may induce stress in WJ-MSCs and impair its function resulting in transdifferentiation of cardiac fibroblasts to myofibroblasts.

To further elucidate whether the impairment of antifibrosis properties can be recovered, we overexpressed anti-fibrosis genes in WJ-MSCs and then co-cultured with $\mathrm{CF}$ under ROS conditions. We hypothesized that administration of MSC-HGF, MSC-IGF1 or MSCSHH might improve anti-fibrotic properties under ROS conditions. The results showed that overexpressedMSC increased the gene expression levels of HGF, IGF1, and SHH even when cultured under ROS conditions. The conditioned media of overexpressedMSC co-cultured with CF showed higher HGF, IGF1 and $\mathrm{SHH}$ protein expression compared to the others. Therefore, overexpression of anti-fibrosis genes; HGF, IGF1, and SHH in MSC may abolish the effect of $\mathrm{H}_{2} \mathrm{O}_{2}$ on anti-fibrosis genes and protein expression. MSCs were then co-cultured with CF. Fibrosis is characterized by the accumulation and deposition of collagen type I and SMA produced from myofibroblasts. Therefore, we investigated fibrogenesis via evaluation of myofibroblast characteristics of $\mathrm{CF}$ during culture. The study was divided into 4 groups as follows; $\mathrm{CF}+\mathrm{MOCK}, \mathrm{CF}+\mathrm{MSC}+\mathrm{H}_{2} \mathrm{O}_{2}, \mathrm{CF}+$ overexpressedMSC, CF+overexpressed-MSC $+\mathrm{H}_{2} \mathrm{O}_{2}$. The results showed that overexpression groups; HGF-MSC, IGF1MSC or SHH-MSC in the presence of $\mathrm{H}_{2} \mathrm{O}_{2}$ were able to decrease the expression of myofibroblastic genes and proteins; COL1 and SMA. These results correlated with previous studies that gene transfer of HGF, IGF1, or $\mathrm{SHH}$ reduced cardiac fibrosis (47). HGF prevents fibrosis via the HGF/Met pathway, which antagonize the TGF- $\beta$ and angiotensin II signaling pathway 
$(38,48)$. Meanwhile, overexpression of IGF1 in cardiomyocytes reduced fibrosis in the mouse model of dilated cardiomyopathy by inhibiting cardiac fibroblast proliferation and reducing the expression of connective tissue growth factor (CTGF), a downstream mediator of the TGF- $\beta$ pathway (49). However, the role of SHH in cardiac fibrosis has not been clearly elucidated. $\mathrm{SHH}$ has been shown to promote fibrosis in some organs such as lung, liver, and kidney via its interaction with the receptor Ptch1/Smo, which activates Gli1, a transcription factor regulating the expression of fibrogenic genes; Snail, COL1, SMA, fibronectin, and desmin. Besides, the SHH pathway may promote epithelial-mesenchymal transition (EMT), which is necessary for tissue fibrosis by induction of TGF- $\beta$ (50). Several studies have suggested that SHH may exert beneficial effects on cardiac repair upon tissue injury. It has been elucidated that SHH is involved in the cardio-protective effect after ischemia via upregulation of VEGF, Ang-1, and Ang-2, which can induce neovascularization and angiogenesis (42).

Taken together, ROS exerted adverse effects on WJ-MSCs by attenuating its anti-fibrotic properties. Our results suggest the overexpression of anti-fibrosis genes can abolish the ROS affected anti-fibrosis properties of WJ-MSCs. Therefore, WJ-MSCs modified to overexpress anti-fibrosis genes could serve as a novel approach for protecting/treating cardiac fibrosis. However, this finding is only a preliminary study, which revealed that the good outcome of stem cell therapy may originate from multiple factors, particularly, the environment of engrafted cells must be a concern and an in vivo study is greatly needed.

\section{Acknowledgements}

This project was supported by Mahidol University, Thailand.

\section{References}

1. Brown RD, Ambler SK, Mitchell MD, Long CS. The cardiac fibroblast: therapeutic target in myocardial remodeling and failure. Annu Rev Pharmacol Toxicol. 2005; 45:657-687.

2. von Harsdorf R, Li PF, Dietz R. Signaling pathways in reactive oxygen species-induced cardiomyocyte apoptosis. Circulation. 1999; 99:2934-2941.

3. Xu Z, Park SS, Mueller RA, Bagnell RC, Patterson C, Boysen PG. Adenosine produces nitric oxide and prevents mitochondrial oxidant damage in rat cardiomyocytes. Cardiovasc Res. 2005; 65:803-812.

4. Tao L, Gao E, Jiao X, Jiao X, Yuan Y, Li S, Christopher TA, Lopez BL, Koch W, Chan L, Goldstein BJ, Ma XL. Adiponectin cardioprotection after myocardial ischemia/ reperfusion involves the reduction of oxidative/nitrative stress. Circulation. 2007; 115:1408-1416.

5. Li L, Zhang Y, Li Y, Yu B, Xu Y, Zhao S, Guan Z. Mesenchymal stem cell transplantation attenuates cardiac fibrosis associated with isoproterenol-induced global heart failure. Transpl Int. 2008; 21:111-1189.

6. Li X, Bai J, Ji X, Li R, Xuan Y, Wang Y. Comprehensive characterization of four different populations of human mesenchymal stem cells as regards their immune properties, proliferation and differentiation. Int J Mol Med. 2014; 34:695-704.

7. Fong CY, Richards M, Manasi N, Biswas A, Bongso A. Comparative growth behaviour and characterization of stem cells from human Wharton's jelly. Reprod Biomed Online. 2007; 15:708-718.

8. Troyer DL, Weiss ML. Wharton's jelly-derived cells are a primitive stromal cell population. Stem Cells. 2008; 26:591-599.

9. Zhou C, Yang B, Tian Y, Jiao H, Zheng W, Wang J, Guan F. Immunomodulatory effect of human umbilical cord Wharton's jelly-derived mesenchymal stem cells on lymphocytes. Cell Immunol. 2011; 272:33-38.

10. Ruan ZB, Zhu L, Yin YG, Chen GC. The mechanism underlying the differentiation of human umbilical cordderived mesenchymal stem cells into myocardial cells induced by 5-azacytidine. Indian J Med Sci. 2010; 64:402-407.

11. Suzuki YJ. Growth factor signaling for cardioprotection against oxidative stress-induced apoptosis. Antioxid Redox Signal. 2003; 5:741-7499.

12. Li LL, Peng C, Zhang M, Liu Y, Li H, Chen H, Sun Y, Zhu C, Zhang Y. Mesenchymal stem cells overexpressing adrenomedullin improve heart function through antifibrotic action in rats experiencing heart failure. Mol Med Rep. 2018; 17:1437-1444.

13. Turtzo LC, Budde MD, Dean DD, Gold EM, Lewis BK, Janes L, Lescher J, Coppola T, Yarnell A, Grunberg NE, Frank JA. Failure of intravenous or intracardiac delivery of mesenchymal stromal cells to improve outcomes after focal traumatic brain injury in the female rat. PloS One. 2015; 10:e0126551.

14. Song H, Cha MJ, Song BW, Kim IK, Chang W, Lim S, Choi EJ, Ham O, Lee SY, Chung N, Jang Y, Hwang KC. Reactive oxygen species inhibit adhesion of mesenchymal stem cells implanted into ischemic myocardium via interference of focal adhesion complex. Stem Cells. 2010; 28:555-563.

15. Zhao W, Zhao T, Chen Y, Ahokas RA, Sun Y. Oxidative stress mediates cardiac fibrosis by enhancing transforming growth factor- $\beta 1$ in hypertensive rats. Mol Cell Biochem. 2008; 317:43-50.

16. Mohammadi Gorji S, Karimpor Malekshah AA, Hashemi-Soteh MB, Rafiei A, Parivar K, Aghdami N. Effect of mesenchymal stem cells on Doxorubicininduced fibrosis. Cell J. 2012; 14:142-151.

17. Zhang GW, Gu TX, Guan XY, Sun XJ, Qi X, Li XY, Wang XB, Lv F, Yu L, Jiang DQ, Tang R. HGF and IGF-1 promote protective effects of allogeneic BMSC transplantation in rabbit model of acute myocardial infarction. Cell Prolif. 2015; 48:661-670.

18. Shams S, Mohsin S, Nasir GA, Khan M, Khan SN. Mesenchymal Stem Cells Pretreated with HGF and FGF4 Can Reduce Liver Fibrosis in Mice. Stem Cells Int. 2015; 747245.

19. Kugler MC, Loomis CA, Zhao Z, Cushman JC, Liu L, Munger JS. Sonic Hedgehog Signaling Regulates Myofibroblast Function during Alveolar Septum Formation in Murine Postnatal Lung. Am J Respir Cell Mol Biol. 2017; 57:280-293. 
20. Thomas NA, Koudijs M, van Eeden FJ, Joyner AL, Yelon D. Hedgehog signaling plays a cell-autonomous role in maximizing cardiac developmental potential. Development. 2008; 135:3789-3799.

21. Paulis L, Fauconnier J, Cazorla O, Thireau J, Soleti R, Vidal B, Ouille A, Bartholome M, Bideaux P, Roubille F, Le Guennec JY, Andriantsitohaina R, Martinez MC, Lacampagne A. Activation of Sonic hedgehog signaling in ventricular cardiomyocytes exerts cardioprotection against ischemia reperfusion injuries. Sci Rep. 2015; 5:7983.

22. Amado LC, Saliaris AP, Schuleri KH, St John M, Xie JS, Cattaneo S, Durand DJ, Fitton T, Kuang JQ, Stewart G, Lehrke S, Baumgartner WW, Martin BJ, Heldman AW, Hare JM. Cardiac repair with intramyocardial injection of allogeneic mesenchymal stem cells after myocardial infarction. Proc Natl Acad Sci U S A. 2005; 102:1147411479 .

23. Lu F, Zhao X, Wu J, Cui Y, Mao Y, Chen K, Yuan Y, Gong D, Xu Z, Huang S. MSCs transfected with hepatocyte growth factor or vascular endothelial growth factor improve cardiac function in the infarcted porcine heart by increasing angiogenesis and reducing fibrosis. Int J Cardiol. 2013; 167:2524-2532.

24. Wang LC, Liu ZY, Gambardella L, Delacour A, Shapiro R, Yang J, Sizing I, Rayhorn P, Garber EA, Benjamin CD, Williams KP, Taylor FR, Barrandon Y, Ling L, Burkly LC. Regular articles: conditional disruption of hedgehog signaling pathway defines its critical role in hair development and regeneration. J Invest Dermatol. 2000; 114:901-908.

25. Pola R, Ling LE, Silver M, Corbley MJ, Kearney M, Blake Pepinsky R, Shapiro R, Taylor FR, Baker DP, Asahara T, Isner JM. The morphogen Sonic hedgehog is an indirect angiogenic agent upregulating two families of angiogenic growth factors. Nat Med. 2001; 7:706-711.

26. Okayama K, Azuma J, Dosaka N, Iekushi K, Sanada F, Kusunoki H, Iwabayashi M, Rakugi H, Taniyama Y, Morishita R. Hepatocyte growth factor reduces cardiac fibrosis by inhibiting endothelial-mesenchymal transition. Hypertension. 2012; 59:958-965.

27. Choe S, Veliceasa D, Bond CW, Harrington DA, Stupp SI, McVary KT, Podlasek CA. Sonic hedgehog delivery from self-assembled nanofiber hydrogels reduces the fibrotic response in models of erectile dysfunction. Acta Biomater. 2016; 32:89-99.

28. Nimsanor N, Phetfong J, Plabplueng C, Jangpatarapongsa K, Prachayasittikul V, Supokawej. A Inhibitory effect of oxidative damage on cardiomyocyte differentiation from Wharton's jelly-derived mesenchymal stem cells. Exp Ther Med. 2017; 14:5329-5338.

29. Dominici M, Le Blanc K, Mueller I, Slaper-Cortenbach I, Marini F, Krause D, Deans R, Keating A, Prockop D, Horwitz E. Minimal criteria for defining multipotent mesenchymal stromal cells. The International Society for Cellular Therapy position statement. Cytotherapy. 2006; 8:315-317.

30. Livak KJ, Schmittgen TD. Analysis of relative gene expression data using real-time quantitative PCR and the $2\left({ }^{-\Delta \mathrm{C}_{\mathrm{T}}} \mathrm{T}\right)$ Method. Method. Methods: A companion to Methods in enzymology. 2001; 25:402-408.

31. Dowling P, Clynes M. Conditioned media from cell lines: A complementary model to clinical specimens for the discovery of disease-specific biomarkers. Proteomics. 2011; 11:794-804.
32. Maurisse R, De Semir D, Emamekhoo H, Bedayat B, Abdolmohammadi A, Parsi H, Gruenert DC. Comparative transfection of DNA into primary and transformed mammalian cells from different lineages. BMC Biotechnol. 2010; 10:9.

33. Watson N, Divers R, Kedar R, Mehindru A, Mehindru A, Borlongan MC, Borlongan CV. Discarded Wharton jelly of the human umbilical cord: A viable source for mesenchymal stromal cells. Cytotherapy. 2015; 17:1824.

34. Rahbarghazi R, Nassiri SM, Khazraiinia P, Kajbafzadeh AM, Ahmadi SH, Mohammadi E, Molazem M, ZamaniAhmadmahmudi M. Juxtacrine and paracrine interactions of rat marrow-derived mesenchymal stem cells, musclederived satellite cells, and neonatal cardiomyocytes with endothelial cells in angiogenesis dynamics. Stem Cells Dev. 2013; 22:855-865.

35. Vinoth KJ, Manikandan J, Sethu S, Balakrishnan L, Heng A, Lu K, Poonepalli A, Hande MP, Cao T. Differential resistance of human embryonic stem cells and somatic cell types to hydrogen peroxide-induced genotoxicity may be dependent on innate basal intracellular ROS levels. Folia Histochem Cytobiol. 2015; 53:169-174.

36. Kim MD, Kim SS, Cha HY, Jang SH, Chang DY, Kim W, Suh-Kim H, Lee JH. Therapeutic effect of hepatocyte growth factor-secreting mesenchymal stem cells in a rat model of liver fibrosis. Exp Mol Med. 2014; 46:e110.

37. Elnakish MT, Kuppusamy P, Khan M. Stem cell transplantation as a therapy for cardiac fibrosis. J Pathol. 2013; 229:347-354.

38. Taniyama Y, Morishita R, Nakagami H, Moriguchi A, Sakonjo H, Shokei K, Matsumoto K, Nakamura T, Higaki J, Ogihara T. Potential contribution of a novel antifibrotic factor, hepatocyte growth factor, to prevention of myocardial fibrosis by angiotensin II blockade in cardiomyopathic hamsters. Circulation. 2000; 102:246-252.

39. Cahill EF, Kennelly H, Carty F, Mahon BP, English K. Hepatocyte Growth Factor Is Required for Mesenchymal Stromal Cell Protection Against Bleomycin-Induced Pulmonary Fibrosis. Stem Cells Transl Med. 2016; 5:1307-1318.

40. Gomez-Mauricio G, Moscoso I, Martin-Cancho MF, Crisostomo V, Prat-Vidal C, Baez-Diaz C, SanchezMargallo FM, Bernad A. Combined administration of mesenchymal stem cells overexpressing IGF-1 and HGF enhances neovascularization but moderately improves cardiac regeneration in a porcine model. Stem Cell Res Ther. 2016; 7:94.

41. Kusano KF, Pola R, Murayama T, et al. Sonic hedgehog myocardial gene therapy: tissue repair through transient reconstitution of embryonic signaling. Nat Med. 2005; 11:1197-1204.

42. Roncalli J, Renault MA, Tongers J, Misener S, Thorne T, Kamide C, Jujo K, Tanaka T, Ii M, Klyachko E, Losordo DW. Sonic hedgehog-induced functional recovery after myocardial infarction is enhanced by AMD3100mediated progenitor-cell mobilization. J Am Coll Cardiol. 2011; 57:2444-2452.

43. Sullivan DE, Ferris M, Pociask D, Brody AR. The latent form of TGF $\beta 1$ is induced by TNFalpha through an ERK specific pathway and is activated by asbestosderived reactive oxygen species in vitro and in vivo. $\mathrm{J}$ Immunotoxicol. 2008; 5:145-149.

44. Liu RM, Gaston Pravia KA. Oxidative stress and 
glutathione in TGF- $\beta$-mediated fibrogenesis. Free Radic Biol Med. 2010; 48:1-15.

45. Coudriet GM, He J, Trucco M, Mars WM, Piganelli JD. Hepatocyte growth factor modulates interleukin-6 production in bone marrow derived macrophages: implications for inflammatory mediated diseases. PloS one. 2010; 5:e15384.

46. Seong GJ, Hong S, Jung SA, Lee JJ, Lim E, Kim SJ, Lee JH. TGF- $\beta$-induced interleukin-6 participates in transdifferentiation of human Tenon's fibroblasts to myofibroblasts. Mol Vis. 2009; 15:2123-2128.

47. Ishikawa H, Jo J, Tabata Y. Liver anti-fibrosis therapy with mesenchymal stem cells secreting hepatocyte growth factor. J Biomater Sci Polym Ed. 2012; 23:22592272.
48. Gallo S, Sala V, Gatti S, Crepaldi T. Cellular and molecular mechanisms of HGF/Met in the cardiovascular system. Clin Sci (Lond). 2015; 129:1173-1193.

49. Touvron M, Escoubet B, Mericskay M, Angelini A, Lamotte L, Santini MP, Rosenthal N, Daegelen D, Tuil D, Decaux JF. Locally expressed IGF1 propeptide improves mouse heart function in induced dilated cardiomyopathy by blocking myocardial fibrosis and SRF-dependent CTGF induction. Dis Model Mech. 2012; 5:481-491.

50. $\mathrm{Hu} \mathrm{L}$, Lin X, Lu H, Chen B, Bai Y. An overview of hedgehog signaling in fibrosis. Mol Pharmacol. 2015; 87:174-182.

(Received July 22, 2019; Revised October 8, 2019; Accepted October 14, 2019) 